\title{
Treatment with intensity-modulated radiation therapy (IMRT) for breast cancer
}

\author{
TrATAMENTO COM RADIOTERAPIA DE INTENSIDADE MODULADA (IMRT) PARA CÂNCER DE MAMA \\ Authorship: Sociedade Brasileira de Radioterapia \\ Participants: Marta GN, Hanna SA, Gadia R \\ Final draft: February 27, 2014
}

http://dx.doi.org/10.1590/1806-9282.60.06.005

The Guidelines Project, an initiative of the Brazilian Medical Association, aims to combine information from the medical field in order to standardize procedures to assist the reasoning and decision-making of doctors.

The information provided through this project must be assessed and criticized by the physician responsible for the conduct that will be adopted, depending on the conditions and the clinical status of each patient.

\section{DESCRIPTION OF THE EVIDENCE COLLECTION METHOD}

Through the development of four relevant clinical questions related with the proposed subject, we tried to present the main evidence for safety, toxicity and effectiveness using different radiotherapy techniques. The study population consisted of female patients of all ages with primary breast cancer treated with radiation therapy to the whole breast, regardless of histological type, staging, context of treatment (radical, adjuvant or palliative) and whether comorbidities were present or not. For this, a systematic review of the literature was performed in primary scientific research databases (Medline - Pubmed; Embase - Elsevier; Lilacs - Bireme; Cochrane Library - Central Register of Controlled Trials). All articles available until July 22, 2013, were included. The search strategy used for Medline research is described in Appendix. Articles were selected based on critical evaluation in search of the best available evidence. Recommendations were prepared based on discussion with the writing group, composed of three members of the Brazilian Society of Radiotherapy. The guideline was reviewed by an independent group specializing in evidence-based clinical guidelines. After completion, the guideline was made available for public consultation for 15 days and the suggestions forwarded to the writers for evaluation and consideration into the final text.

\section{Grade Of Recommendation AND STRENGTH OF EVIDENCE}

A. Experimental or observational studies of higher consistency.
B. Experimental or observational studies of lower consistency.

C. Case reports (non-controlled studies).

D. Opinions without critical evaluation, based on consensus, physiological studies, or animal models.

\section{Objective}

To assess the most appropriate method of radiation therapy for treating patients with primary breast tumors.

\section{Conflict of interest}

No conflict of interest informed.

\section{INTRODUCTION}

Breast cancer is the most common malignancy (except for non-melanoma skin tumors) and the leading cause of cancer death among women(D). ${ }^{1}$ According to data from the National Cancer Institute, in Brazil, for the year 2012, 52,680 new cases were estimated, which corresponds to an estimated risk of 52 new cases per 100,000 women(D). ${ }^{2}$

Radical mastectomy, as proposed by Halsted in 1894, has been the standard treatment for breast cancer regardless of any associated factor. However, in the last 50 years, there has been a change in surgical approach, following the trend of breast conservation, whilst continuing to offer oncological safety. According to this rationale, adjuvant radiotherapy (RT) is fundamental to treat all patients with initial in situ and invasive tumors who undergo conservative surgery, as well as those with locally advan- 
ced disease (tumor size $>5 \mathrm{~cm}$, more than three lymph nodes affected, and more).

From the standpoint of the existing RT techniques, conventional planning (2D) is performed based on two dimensions (planar images such as X-ray) with dose distribution calculation in a single plane (contour) of the patient. The total volume irradiated is not considered, that is, the dose variation outside this contour is ignored (no accurate volumetric measurement of dose distribution). The combinations of the radiation beams are simple and there is a need for good margin coverage, in order to ensure complete coverage of the treatment target volume. Thus, critical structures (organs at risk) are often included in the treatment field, which contributes greatly to increased toxicity.

In the late 1980s, with the improvement of hardware and planning systems, it was possible to provide tools capable of assisting the evaluation of treatments that have become fully three-dimensional (conformal technique - 3D). In 3D RT for the breast, dose distribution is calculated over the total volume irradiated, with tissue inhomogeneity corrections, considering the contour of the patient's chest at different levels. The measurements of heart volume and lung volume, which are irradiated through field entries, are known for the calculation of dose-volume histograms. This is an important tool because it helps to assess the homogeneity of planning, as well as measures the dose to be received by each organ during treatment delivery. Ultimately, it is the tool used to determine whether a treatment is prohibitive due to the high chance of side effects, or if it is acceptable on account of greater sparing effect for the organs at risk.

As previously said, 3D RT offers better distribution of the prescribed dose at the target volume. However, dose deposition in tissues using the $3 \mathrm{D}$ technique is practically the same as in 2D RT. Thus, in the scope of treatment, the intensity of radiation is the same in each treatment beam, that is, the dose is uniformly delivered to both the tumor and normal adjacent tissues in the treatment target area.

In order to improve the intensity of non-uniform beams, a technique of beam intensity-modulated RT (IMRT) was developed. For this, an inverse planning is used, consisting in first defining the organs at risk and target volumes, and the dose that each structure should receive. As a result, a non-uniform deposition of dose is obtained within treatment area, which is achieved by dividing the field into several sub-fields. In the case of breast cancer, in which the incidence of treatment beams is arranged tangentially to the chest wall, conventional beams end up generating inhomogeneous dose distributions in the breast, particularly in areas of lower thickness. Therefore, the treated area lacks dose homogenization, while IMRT, which usually allows a 'non-uniform deposition' of the dose at the treatment target, offers exactly the opposite: the homogenization of the dose in the breast.

Importantly, in practice, for the treatment of breast cancer with radiotherapy, the so-called IMRT involves the adoption of two distinct strategies:

1. the use of inverse planning, as mentioned above;

2. the use of a computerized planning system capable of evaluating the dose distribution in the overall volume. Through visualization of high-dose regions, field segmentation is carried out manually using collimation blocks or a multileaf collimator, resulting in a more homogeneous dose distribution (no need to use reverse planning).

\section{IS DOSE DISTRIBUTION FOR BREAST IRRADIATION SUPERIOR WITH INTENSITY- MODULATED RADIATION THERAPY (IMRT) COMPARED TO CONVENTIONAL AND CONFORMAL RADIOTHERAPY?}

IMRT is the modality that provides best dose coverage at the treatment target (breast) compared to the conformal and conventional techniques $(\mathbf{B}){ }^{3,4}$

In addition, IMRT significantly reduces the dose delivered to organs at risk. The dose reduction in the contralateral breast can reach $50 \%$, which reduces the likelihood of radiation-induced breast cancer especially in young women $(\mathbf{B}){ }^{5,6}$ The same was noted with regard to other structures such as the heart and lungs (ipsilateral and contralateral), which can be associated with a reduced risk for chronic lung and heart disease $(\mathbf{B}) .^{7-14}$ This benefit was also demonstrated in patients undergoing mastectomy and adjuvant radiation therapy for plastron $(\mathbf{B}){ }^{7}$

\section{Recommendation}

Intensity-modulated radiation therapy (IMRT) is a modality that provides better dose distribution in breast irradiation. 


\section{IS IT LESS TOXIC TO USE INTENSITY-MODULATED RADIATION THERAPY (IMRT) COMPARED TO CONVENTIONAL OR CONFORMAL RADIATION THERAPY FOR PRIMARY BREAST TUMORS?}

Toxicity related to radiation therapy can be temporally divided into two sub-categories: acute and delayed.

When comparing IMRT with conventional or conformal radiation therapy of the breast, two studies had acute toxicity as the main outcome.

A phase III multicenter study including 358 patients showed that the rates of moist desquamation during radiotherapy and up to six weeks after treatment were $31.2 \%$ with IMRT, and $47.8 \%$ with conventional or conformal radiation therapy $(\mathrm{p}=0.002)(\mathbf{A}){ }^{16}$

A recently published English study with median follow-up of five years and which included 1,145 treated patients showed that the use of IMRT reduces the rates of telangiectasia (OR 0.58, 95\%CI: $0.36-0.92, \mathrm{p}=0.021)(\mathbf{A})^{3}(\mathbf{B}) .^{17}$

Regarding delayed toxicity, a minimum of five years for patient follow-up is required. Some institutional series reported a low rate of delayed toxicity related to radiation therapy: a study with 7.5 years of median follow-up reports rates of delayed skin toxicity grade II or higher at 39 vs. $52 \%(\mathrm{p}=0.004)$ with IMRT and conventional radiotherapy, respectively $(\mathbf{D}) .{ }^{18}$ Rates of pneumonitis, lymphoedema, and tumor recurrence within the follow-up were not statistically different between the two groups $(\mathbf{D}) .^{18}$ A similar study showed after 4.7 years of median follow-up that the use of IMRT reduces the rates of acute and delayed toxicity, with respective rates of delayed breast edema grade II or higher at 6 versus $1 \%(\mathrm{p}=0.009)(\mathbf{B}) .{ }^{19}$ For patients with larger breasts (volume $>1,600 \mathrm{~cm}^{3}$ ), the gains were more expressive with IMRT: chronic breast edema ( 3 vs. $30 \%$, $\mathrm{p}=0.007)$ and hyperpigmentation ( 3 vs. $41 \%, \mathrm{p}=0.001)(\mathbf{B}) .{ }^{19}$

\section{Recommendation}

There is less acute toxicity with the use of intensity-modulated radiation therapy (IMRT) compared to conventional or conformal radiation therapy for primary breast tumors, especially regarding the rate of moist desquamation during radiotherapy. Delayed skin toxicity is less prevalent with IMRT, with a lower incidence of chronic breast edema, hyperpigmentation, and telangiectasia. These gains are also seen in larger breasts treated with this technique.

IS THE QUALITY OF LIFE AFFECTED TO THE POINT OF JUSTIFYING THE USE OF INTENSITY-MODULATED RADIATION THERAPY (IMRT) OVER CONFORMAL OR CONVENTIONAL RADIATION THERAPY?

Quality of life is a difficult outcome to be measured, with many uncertainties, including the difficulty in defining it and the subjectivity of measurements. For the treatment of breast cancer, quality of life was assessed in studies using specific tools such as questionnaires or through cosmetic evaluation of patients, which is intrinsically linked to quality of life and personal satisfaction.

Cosmesis was evaluated in two randomized studies as the primary outcome according to the use of IMRT.

306 women underwent cosmetic assessment by serial photographs after 1, 2 and 5 years of follow-up. The rate of change in the appearance of the breast was $58 \mathrm{vs}$. $40 \%(\mathrm{p}=0.008)$ for conventional radiotherapy and IMRT, respectively $(\mathbf{B}){ }^{4}$

The findings illustrated a $68 \%$ reduction in the risk of worsening of cosmesis using the RTOG scale after IMRT $(\mathrm{OR}=0.68 ; 95 \% \mathrm{CI}, 0.48$ to $0.96 ; \mathrm{p}=0.027)(\mathbf{B})^{3}(\mathbf{A}) .^{17}$

Direct evaluation of the quality of life through scores or questionnaires was carried out in the study, which assessed patients based on two questionnaires: EORTC Quality of Life Questionnaire C-30 general module and BR-23 module self-assessment. During patient follow-up, there was a significant correlation between the two scores for quality of life and the presence of breast pain and radiodermitis $(\mathbf{A}) \cdot{ }^{16}$

\section{Recommendation}

The use of intensity-modulated radiation therapy (IMRT) reduces the risk of worsening of cosmesis. There is no evidence for other outcomes related to quality of life.

\section{Are there differences in EFFECtiveness, LOCAL CONTROL OR OVERALL SURVIVAL AMONG THE TECHNIQUES OF INTENSITY-MODULATED RADIATION THERAPY (IMRT), CONFORMAL RT AND CONVENTIONAL RT?}

In three phase III randomized trials comparing conventional or conformal RT with IMRT, effectiveness, local control and overall survival were not the main outcomes evaluated. The reason for this is that the hypothesis was not proposed because the prescribed doses and irradiated volumes were equal, regardless of the technique. In one of these studies, with five years of median follow up, the rate of local control in the group of women treated with IMRT and conventional RT in five years was 98.6 and $97.4 \%$, respectively $(\mathrm{p}=0.36)$ and the overall survival in five years was 92.5 and $91.7 \%$, respectively $(\mathrm{p}=0.88)(\mathbf{A}) .{ }^{17}$

\section{Recommendation}

IMRT compared with conventional or conformal radiation therapy techniques for the breast has benefits in terms of dosage, toxicity and quality of life, while it does not affect local control and overall survival of patients. 


\section{ApPendix}

Medline search strategy

(Breast Neoplasms [Mesh] OR Breast Neoplasm OR Neoplasm, Breast OR Neoplasms, Breast OR Tumors, Breast OR Breast Tumors OR Breast Tumor OR Tumor, Breast OR Mammary Carcinoma, Human OR Carcinoma, Human Mammary OR Carcinomas, Human Mammary OR Human Mammary Carcinomas OR Mammary Carcinomas, Human OR Human Mammary Carcinoma OR Mammary Neoplasms, Human OR Human Mammary Neoplasm OR Human Mammary Neoplasms OR Neoplasm, Human Mammary OR Neoplasms, Human Mammary OR Mammary Neoplasm, Human OR Breast Cancer OR Cancer, Breast OR Cancer of the Breast OR Cancer of Breast) AND (Radiotherapy, Intensity-Modulated [Mesh] OR Intensity-Modulated Radiotherapies OR Intensity-Modulated Radiotherapy OR Radiotherapies, Intensity-Modulated OR Radiotherapy, Intensity Modulated OR IMRT OR Volumetric-Modulated Arc Therapy OR Arc Therapies, Volumetric-Modulated OR Arc Therapy, Volumetric-Modulated OR Therapies, Volumetric-Modulated Arc OR Therapy, Volumetric-Modulated Arc OR Volumetric Modulated Arc Therapy OR Volumetric-Modulated Arc Therapies OR Intensity-Modulated Arc Therapy OR Arc Therapies, Intensity-Modulated OR Arc Therapy, Intensity-Modulated OR Intensity Modulated Arc Therapy OR Intensity-Modulated Arc Therapies OR Therapies, Intensity-Modulated Arc OR Therapy, Intensity-Modulated Arc OR Helical Tomotherapy OR Helical Tomotherapies OR Tomotherapies, Helical OR Tomotherapy, Helical) AND (Radiotherapy, Conformal [Mesh] OR Conformal Radiotherapies OR Radiotherapies, Conformal OR Conformal Radiotherapy OR Radiotherapy Planning, Computer-Assisted OR Radiotherapy Dosage OR Radiotherapy Planning, Computer-Assisted OR 3D radiotherapy OR tridimensional radiotherapy OR 3D RT OR 3DRT OR 3DCRT OR CRT OR 3D-CRT) OR (Conventional radiotherapy OR 2D radiotherapy OR 2D RT OR 2DRT OR 2D-CRT OR standard radiotherapy $O R$ two-dimensional radiotherapy).

\section{Other guidelines at www.projetodiretrizes.org.br}

\section{References}

1. Parkin DM, Bray F, Ferlay J, Pisani P. Global cancer statistics, 2002. CA Cancer J Clin 2005; 55:74.

2. Ministério da Saúde do Brasil. Estimativas para o ano de 2012 de número de casos novos e de óbitos por câncer em mulheres, segundo localização pri- mária. Access on 7/22/2013. Available from: http://www.inca.gov.br/estimativa/2012/index.asp?ID $=5$

3. Barnett GC, Wilkinson J, Moody AM, Wilson CB, Sharma R, Klager S, Hoole ACF, Twyman N, Burnet NG, Coles CE. A randomised controlled trial of forward-planned radiotherapy (IMRT) for early breast cancer: Baseline characteristics and dosimetry results. Radiother Oncol 2009;92:34-41.

4. Donovan E, Bleakley N, Denholm E, et al. Randomised trial of standard 2D radiotherapy (RT) versus intensity modulated radiotherapy (IMRT) in patients prescribed breast radiotherapy. Radiother Oncol 2007;82:254-64.

5. Burmeister J, Alvarado N, Way S, McDermott P, Bossenberger T, Jaenisch $\mathrm{H}$, Patel R, Washington T. Assessment and minimization of contralateral breast dose for conventional and intensity modulated breastradiotherapy. Med Dosim 2008;33:6-13.

6. Bhatnagar AK, Brandner E, Sonnik D, Wu A, Kalnicki S, Deutsch M, Heron DE. Intensity modulated radiation therapy (IMRT) reduces the dose to the contralateral breastwhen compared to conventional tangential fields for primary breast irradiation. Breast Cancer Res Treat 2006;96:41-6.

7. Landau D, Adams EJ, Webb S, Ross G. Cardiac avoidance in breast radiotherapy:a comparison of simple shielding techniques with intensity-modulatedradiotherapy. Radiother Oncol 2001;60:247-55.

8. Johansen S, Cozzi L, Olsen DR. A planning comparison of dose patterns in organs at risk and predicted risk for radiation induced malignancy inthe contralateral breast following radiation therapy of primary breast using conventional, IMRT and volumetric modulated arc treatment techniques. Acta Oncol. 2009;48:495-503.

9. Stillie AL, Kron T, Herschtal A, Hornby C, Cramb J, Sullivan K, Chua B. Does inverse-planned intensity-modulated radiation therapy have a role in the treatment of patients with left-sidedbreast cancer? J Med Imaging Radiat Oncol 2011;55:311-9.

10. Ercan T, Idem S, Alço G, Zengin F, Atilla S, Dinçer M, Okkan S. Dosimetric comparison of field in field intensity-modulated radiotherapy technique with conformal radiotherapy techniques in breast cancer. Jpn J Radiol 2010;28:283-9.

11. Fong A, Bromley R, Beat M, Vien D, Dineley J, Morgan G. Dosimetric comparison of intensity modulated radiotherapy techniques and standard wedged tangents for whole breast radiotherapy. J Med Imaging Radiat Oncol 2009;53:92-9.

12. Schubert LK, Gondi V, Sengbusch E, Westerly DC, Soisson ET, Paliwal BR, Mackie TR, Mehta MP, Patel RR, Tomé WA, Cannon GM. Dosimetric comparison of leftsided whole breast irradiation with 3DCRT, forward-planned IMRT, inverse-plannedIMRT, helical tomotherapy, and topotherapy. Radiother Oncol 2011;100:241-6.

13. Baycan D, Karacetin D, Balkanay AY, Barut Y. Field-in-field IMRT versus 3D-CRT of the breast. Cardiac vessels, ipsilateral lung, and contralateral breast absorbed doses in patients with left-sided lumpectomy: a dosimetric comparison. Jpn J Radiol 2012;30:819-23.

14. Coon AB, Dickler A, Kirk MC, Liao Y, Shah AP, Strauss JB, Chen S, Turian J, Griem KL. Tomotherapy and multifield intensity-modulated radiotherapy planning reduce cardiac doses in left-sided breastcancer patients with unfavorable cardiac anatomy. Int J Radiat Oncol Biol Phys 2010;78:104-10.

15. Rudat V, Alaradi AA, Mohamed A, Ai-Yahya K, Altuwaijri S. Tangential beam IMRT versus tangential beam 3D-CRT of the chest wall in postmastectomy breast cancerpatients: a dosimetric comparison. Radiat Oncol 2011;06:26 AM.

16. Pignol JP, Olivotto I, Rakovitch E, et al. A multicenter randomized trial of breast intensity-modulated radiation therapy to reduce acute radiation dermatitis. J Clin Oncol 2008;26:2085-92.

17. Mukesh MB, Barnett GC, Wilkinson JS, Moody AM, Wilson C, Dorling L, Chan Wah Hak C, Qian W, Twyman N, Burnet NG, Wishart GC, Coles CE. Randomized Controlled Trial of Intensity-Modulated Radiotherapy for Early Breast Cancer: 5-Year Results Confirm Superior Overall Cosmesis. J Clin Oncol. 2013 Sep 16. [Epub ahead of print]

18. Mcdonald MW, Godette KD, Butker EK, Davis LW, Johnstone PAS. Long -Term Outcomes of IMRT for Breast Cancer: A Single-Institution Cohort Analysis. Int J Radiat Oncol Biol Phys 2008; 72:1031-1040.

19. Harsolia A, Kestin L, Grills I, Wallace M, Jolly S, Jones C, Lala M, Martinez A, Schell S, Vicini FA. Intensity-Modulated Radiotherapy Results in Significant Decrease in Clinical Toxicities Compared with Conventional Wedge-Based Breast Radiotherapy. Int J Radiat Oncol Biol Phys 2007;68:1375-1380. 